(C) World Scientific Publishing Company

\title{
Nuclear Theory and Science of the Facility for Rare Isotope Beams
}

त

\author{
A.B. BALANTEKIN \\ Department of Physics, University of Wisconsin-Madison, Wisconsin 53706, USA \\ J. CARLSON \\ Theoretical Division, Los Alamos National Laboratory, Los Alamos, New Mexico 87545, USA \\ D.J. DEAN \\ Physics Division, Oak Ridge National Laboratory, Oak Ridge, Tennessee 37831, USA \\ G.M. FULLER \\ Department of Physics, University of California, San Diego, La Jolla, California 92093-0319 \\ R.J. FURNSTAHL \\ Department of Physics, The Ohio State University, Columbus, Ohio 43210, USA

\section{HJORTH-JENSEN} \\ National Superconducting Cyclotron Laboratory and Department of Physics and Astronomy \\ Michigan State University, East Lansing, Michigan 48824, USA \\ R.V.F. JANSSENS \\ Physics Division, Argonne National Laboratory, Argonne, Illinois 60439, USA

\section{BAO-AN LI} \\ Department of Physics $\&$ Astronomy, Texas A\&M University-Commerce \\ Commerce, Texas 75429, USA

\section{W. NAZAREWICZ} \\ Department of Physics \& Astronomy, University of Tennessee, Knoxville, Tennessee 37996, \\ $U S A$ \\ Physics Division, Oak Ridge National Laboratory, Oak Ridge, Tennessee 37831, USA \\ witek@utk.edu

\section{F.M. NUNES}

National Superconducting Cyclotron Laboratory and Department of Physics and Astronomy 
W.E. ORMAND

Physics Division, Lawrence Livermore National Laboratory, Livermore, California 94551, USA

S. REDDY

Institute for Nuclear Theory, University of Washington, Seattle, Washington 98195, USA

\title{
B.M. SHERRILL
}

National Superconducting Cyclotron Laboratory and Department of Physics and Astronomy, Michigan State University, East Lansing, Michigan 48824, USA

\author{
Received (Day Month Year)
}

Revised (Day Month Year)

\begin{abstract}
The Facility for Rare Isotope Beams (FRIB) will be a world-leading laboratory for the study of nuclear structure, reactions and astrophysics. Experiments with intense beams of rare isotopes produced at FRIB will guide us toward a comprehensive description of nuclei, elucidate the origin of the elements in the cosmos, help provide an understanding of matter in neutron stars, and establish the scientific foundation for innovative applications of nuclear science to society. FRIB will be essential for gaining access to key regions of the nuclear chart, where the measured nuclear properties will challenge established concepts, and highlight shortcomings and needed modifications to current theory. Conversely, nuclear theory will play a critical role in providing the intellectual framework for the science at FRIB, and will provide invaluable guidance to FRIB's experimental programs. This article overviews the broad scope of the FRIB theory effort, which reaches beyond the traditional fields of nuclear structure and reactions, and nuclear astrophysics, to explore exciting interdisciplinary boundaries with other areas.
\end{abstract}

Keywords: Nuclear Structure and Reactions. Nuclear Astrophysics. Fundamental Interactions. High Performance Computing. Rare Isotopes. Radioactive Beams.

PACS Nos.: 21.60.-n, 21.30.-x, 21.65.-f, 24.10.-i, 24.80.+y, 25.60.-t, 26.30.-k, 26.50.+x, 26.60.-c

\section{Introduction: New Opportunities with FRIB}

Nuclear physics plays a key role in our quest to understand the Universe $\sqrt{12}$ In recent years, researchers have made remarkable progress in our fundamental understanding of the complex and fascinating system that is the nucleus $\frac{1314}{4}$ This progress has been driven by new theoretical insights and increased computational power, as well as by experimental access to new isotopes with a large excess of neutrons or protons. However, while much has been learned so far about nuclear systems and associated phenomena, much remains to be understood.

The Facility for Rare Isotope Beams (FRIB) $\frac{5}{5}$ which is a sponsored project of the US Department of Energy, Office of Science, will push the frontiers of nuclear science by providing access to the widest range of isotopes possible. For example, to explore changes in shell structure, useful yields of nickel isotopes will be available from ${ }^{48} \mathrm{Ni}$ to ${ }^{84} \mathrm{Ni}$, thereby spanning the full range of neutrons in the $p f g$-shells. The 
key is for the facility to deliver very high power heavy-ion primary beams, ${ }^{6} 400 \mathrm{~kW}$ minimum, at energies of at least $200 \mathrm{MeV} / \mathrm{u}$ to be used in the production of rare isotopes. ${ }^{7}$ These high-power beams will be generated by a superconducting linear accelerator coupled with a production area designed to operate at high current 899 The broad scientific program requires rare-isotope beams at energies ranging from stopped ions in traps $\frac{10}{10}$ to ions at relativistic energies of hundreds of $\mathrm{MeV} / \mathrm{u}$. FRIB will have these capabilities by using full-energy beams following in-flight separation, stopped ions thermalized using a variety of ion catcher schemes, and reaccelerated ions delivered by the ReA superconducting linear post-accelerator ${ }^{9}$ The goal is to reach reaccelerated beam energies of $12-20 \mathrm{MeV} / \mathrm{u}$. As a result, the full complement of direct reactions (including high- $\ell$ transfer and deep-inelastic reactions) will be accessible for experimentation. An advantage of the in-flight production and reacceleration approach is that isotopes of all elements will normally be available with very short development times and high efficiency (approaching 10 to 20\%). The technique will also provide beams of most isotopes, even those with short (tens of ms) half-lives. This offers the possibility to perform experiments, for example, with beams of highly refractory elements along the $N=126$ line of isotones below ${ }^{208} \mathrm{~Pb}$, as required to improve $r$-process nucleosynthesis models.

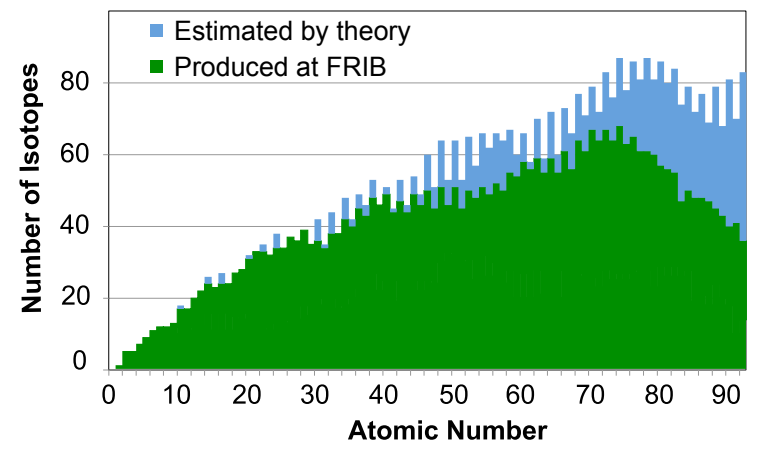

Fig. 1. Number of isotopes of elements up to $Z=92$ estimated to be produced in sufficient quantities at FRIB (green bars) to allow study of their structure and determine at least one property other than simple observation. The number of isotopes estimated to exist (blue bars) is taken from the recent theoretical survey of Ref. 11 FRIB is predicted to produce nearly $80 \%$ of all possible isotopes in this range.

The anticipated range of isotopes to be available at FRIB, as estimated using the LISE ${ }^{++}$program, 12 is shown in Fig. 1. It is compared to the predicted number of possible isotopes from the average of the Density Functional Theory (DFT) predictions 11 With the $400 \mathrm{~kW}$ beams of FRIB, nearly $80 \%$ of all isotopes of elements up to uranium may become available for study. This includes many new isotopes estimated to lie along the drip lines (perhaps even up to element $Z=61$ as shown in the figure) and many nuclei with skins predicted to be greater than $0.5 \mathrm{fm}$ (cf. Ref. 13). In addition, the facility will have provisions to collect unused 
isotopes and make them available for experiments and applications in other fields, such as medicine ${ }^{14}$ One option being considered is to collect isotopes produced in the beam dump by uranium stopping in water. This approach would, for example, make nearly continuous supplies of ${ }^{223} \mathrm{Rn}$ or ${ }^{225} \mathrm{Ra}$ available for fundamental interaction studies searching for an atomic electric dipole moment 15 Other options are to provide a source of isotopes such as ${ }^{67} \mathrm{Cu}$ or ${ }^{149} \mathrm{~Tb}$ for medical studies ${ }^{14}$

In this broad overview of FRIB theory, we concentrate on major themes pertaining to science, organization, and education aspects of the effort. The references and links cited are not meant to be inclusive; they should rather be used as sources to further in-depth information. This paper is organized as follows. Section 2 summarizes the science case for FRIB, in the context of the overarching science questions. The interdisciplinary aspects of the FRIB program are presented in Sec. 3 . Section 4 discusses various ways of enhancing the coupling between experiment and theory. High performance computing will play a key role in FRIB science program; this is discussed in Sec. 5. Sections 6 and 7 talk about organization of the FRIB theory community and educational aspects, respectively. Finally, Sec. 8 offers a broad perspective on FRIB theory.

\section{FRIB Science Overview}

The science case for FRIB has been formulated over many years and is well documented $\sqrt{12 \mid 4}$ In short, the facility will address - on many levels - the overarching science questions identified by the U.S. National Academy of Science in the fourth decadal survey of nuclear physics entitled Exploring the Heart of Matter ${ }^{1}$ 1) How did matter come into being and how does it evolve? 2) How does subatomic matter organize itself and what phenomena emerge? 3) Are the fundamental interactions that are basic to the structure of matter fully understood?, and 4) How can the knowledge and technological progress provided by nuclear physics best be used to benefit society? Together with experiment, future developments in low-energy nuclear theory and computational science will be critical in answering these questions.

The origin of the elements. Our radioactive galaxy demonstrates continuing formation of new short-lived elements by nuclear reaction sequences (see Fig. 2 and Refs. (16, 19). Nuclear structure helps dictate galactic chemical evolution. One example is the neutron driven $s$ - and $r$-processes, responsible for building heavy elements. The resulting final abundances of these processes reflect nuclear shell structure, which gives rise to the respective nucleosynthesis paths. Another example concerns the $r p$-process, which provides a sensitive probe for neutron star surfaces and crusts. To this day we do not know exactly where the heavy elements were made. Possible r-process sites include supernovae and neutron star mergers. Differences in reaction paths, which depend on the masses and lifetimes of the nuclei involved, could affect abundance signatures for each site. Theory is a key component to resolve this mystery. Indeed, nuclear models provide structural input for key nuclei not accessible to experiment that participate in reaction networks, and large scale computational 
simulations - such as those shown in Fig. 2 bottom - tell us about astrophysical conditions at possible sites.

The theory roadmap includes deriving nuclear interactions from QCD and connecting those to the structure of the lightest elements and Big Bang nucleosynthesis. The combined effort of new experiments and theoretical/computational approaches will enable us to accurately determine all relevant properties and reactions of light nuclei, in particular neutron-rich nuclei formed during stellar evolution. Interactions obtained from effective theories of QCD, density functional theories, experiments, and astrophysical observations will describe the properties of nucleonic matter found in nature: the nuclear landscape, neutron stars and supernovae.

While great progress has been made in the last decade in the theoretical descrip-
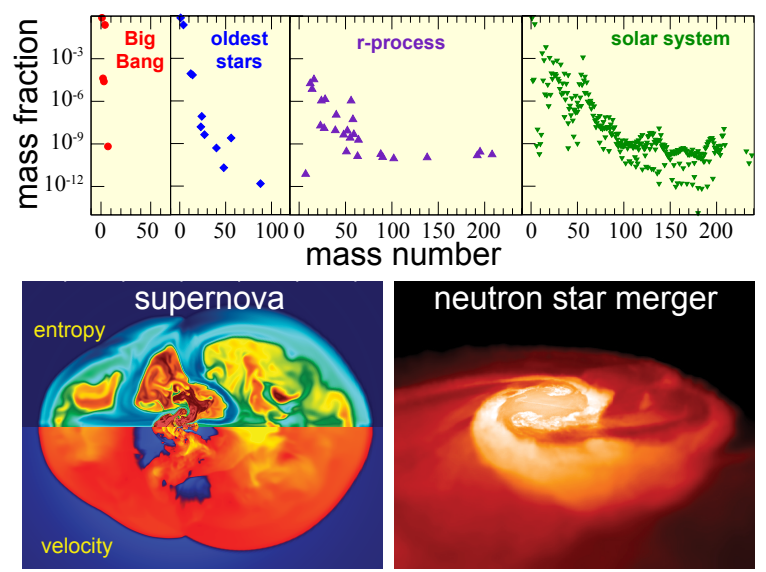

Fig. 2. Top: Understanding the observed sequence of abundance enrichment of nuclides $\sqrt{16}$ is a challenge to theory. Bottom: Advanced simulations of supernovd ${ }^{17}$ (left) and neutron star

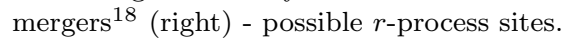

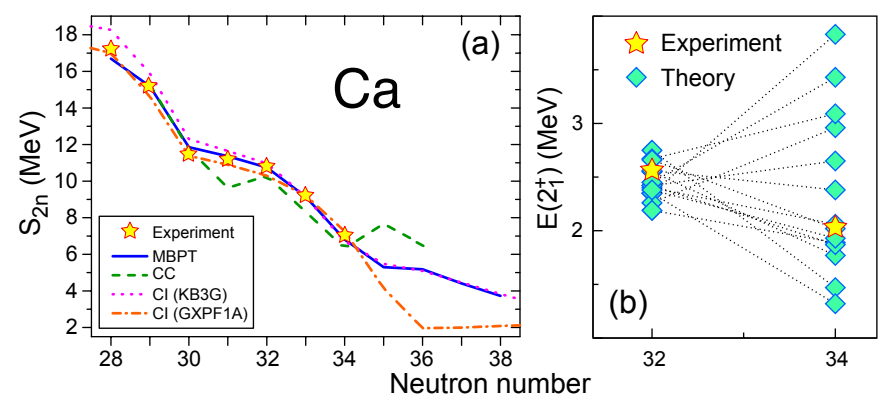

Fig. 3. Theoretical predictions for (a) two-neutron separation energies $S_{2 n}$ and (b) $2_{1}^{+}$excitations (insert) in calcium isotopes compared to experiment 20|21|22 and in nuclei yet to be measured. Theoretical models shown include: Many Body Perturbation theory (MBPT), coupled cluster (CC) approach, and configuration interaction (CI) methods with different effective interactions (cf. Refs. 21, 22 for details). 
tion of nuclear structure by $a b$ initio methods, configuration interaction approaches, and nuclear density functional theory, the exploration of neutron-rich systems is still in its infancy. Figure 3 provides theory predictions for the neutron-rich calcium isotopes, which are a frontier for probing nuclear forces and shell structure. Predictions for masses (by way of two-neutron separation energies) show good agreement for measured nuclei, but diverge where not yet constrained by experiment $20 \mid 21$ This divergence is especially evident for the $2_{1}^{+}$excitation energies ${ }^{22}$ The interplay between theory and experiment at FRIB will lead to a robust phenomenology with controlled and quantitative uncertainties for the theory predictions of unmeasured nuclei, see Sec. 4 for more discussion.

Organization of sub-atomic matter. The nature of nuclear forces and the mechanism of nuclear binding produce amazingly regular patterns in nuclei. Theory provides the framework to understand the emergence of these collective phenomena.

Finite nuclei exhibit phase-transitional behavior, critical points as a function of particle number, spin, and temperature. To understand what causes the emergent phenomena in atomic nuclei, we need predictive models of small- and largeamplitude collective motion, such as those involved in fission and heavy-ion fusion. The atomic nucleus is an open quantum system ${ }^{23}$; hence, phenomena of nuclear structure are intimately connected to reactions, and both should be described in a unified way. There are challenges in achieving this unified description: the inclusion of the particle(s) continuum and its impact on properties of weakly bound states (such as halos) and unbound nuclear states, and understanding the role of reaction thresholds on the appearance of collective cluster states.

Extended nucleonic matter is another avenue to understand emergent phenomena. Only with theory can we explore the connection between neutron-rich matter in the Cosmos and in the laboratory. Figure 4 illustrates the multi-disciplinary nature of this quest. Answers to many challenging scientific questions ranging from the dynamics of supernova and heavy-ion collisions to the structure of neutron stars and rare isotopes, all depend critically on the Equation of State (EOS) of neutron-rich nucleonic matter. Fig. 4 demonstrates that the isospin-asymmetric part of the EOS, namely the density dependence of nuclear symmetry energy, is still not fully understood. As seen in Fig. 4 (a), while significant effort has been devoted to constraining the symmetry energy parameters around the saturation density from the data obtained in terrestrial laboratories, astrophysical observations, and nuclear theory, large uncertainties still remain $\sqrt[24 \mid 26]{ }$ Of particular importance is the determination of the symmetry energy at supra-saturation densities.

Figure 4(b) (from Ref. 25) displays the mass-radius relation for a neutron star as predicted by various theoretical models. The typical mass of a neutron star is about 1.4 solar masses, and the typical radius is thought to be about $12 \mathrm{~km}$. One of the main science drivers of FRIB is the study of nuclei with neutron skins three or four times thicker than is currently possible. Studies of neutron skins in heavy nuclei and investigations of high-frequency nuclear oscillations and intermediate energy nuclear reactions with a range of proton and neutron-rich nuclei will help pin down 

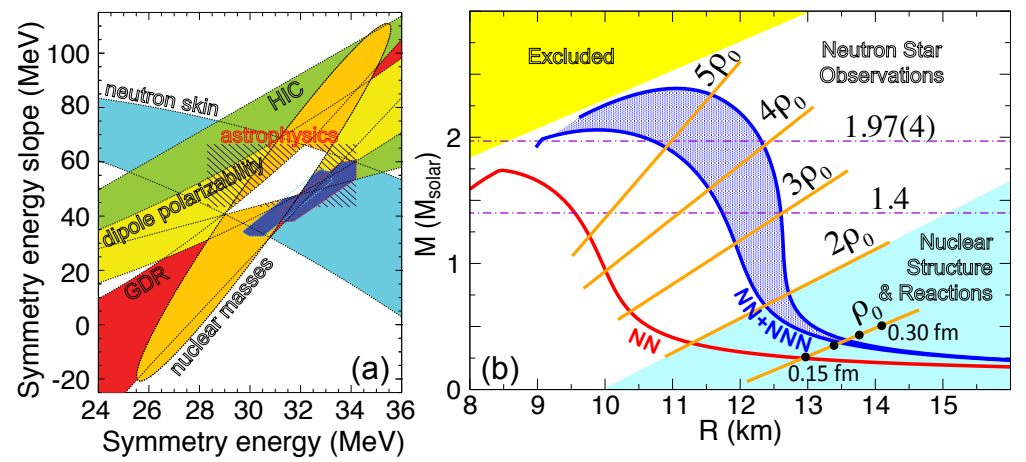

Fig. 4. Left: Summary of constraints on symmetry energy parameters ${ }^{24}$ The experimental constraints from nuclear data (masses, neutron skin thicknesses, dipole polarizability of ${ }^{208} \mathrm{~Pb}$, giant dipole resonances (GDR), and isotope diffusion in heavy-ion collisions (HIC) are marked. The hatched rectangle shows constraints from fitting astrophysical observations. The two closed regions show neutron matter constraints. The enclosed white area is the experimentally-allowed overlap region. Right: Predicted relation between mass and radius of a neutron star modeled with forces involving two nucleons (NN) and forces also involving three nucleons (NN+NNN) 25 The three-nucleon forces are both essential and poorly known, as indicated by a dark blue uncertainty band. The orange lines roughly indicate the predicted central density of the neutron star. The black dots mark the predicted values of the neutron skin in ${ }^{208} \mathrm{~Pb}$. The accurate measurement of a large neutron star mass $\mathrm{M}=1.97(4) M_{\text {solar }}$ provides a strong constraint on theoretical models.

the behavior of nuclear matter at densities below twice the typical nuclear density $\rho_{0}$. At higher densities, relativity and the observations of a nearly two-solar-mass neutron stars ${ }^{27 / 28}$ place severe constraints on the relationship between the pressure and density of nuclear matter.

Fundamental symmetries. Experimental tests of the Standard Model using the nucleus as a laboratory include: searches of atomic electric dipole moments $(\mathrm{EDM})$ in rare isotopes; parity violation tests in Fr; CKM matrix unitarity tests by superallowed $\beta$ decay measurements in $N \approx Z$ nuclei; and searches of exotic scalar and tensor couplings in $\beta$ decay.

Here again, theory and experiment go hand in hand. A variety of nuclearstructure calculations are critical to tests of the Standard Model. These include the isospin-mixing corrections in superallowed $\beta$ decays; nuclear anapole moments in parity violation; Schiff moments for atomic EDM searches; ordinary and neutrinoless double- $\beta$ decay matrix elements, and comparison with observables. These theory predictions are typically needed with high accuracy and quantified uncertainties. In addition, new weak interaction signatures need to be explored to probe astrophysical environments. For example, neutrino signatures probe the burning conditions and chemistry of the solar core and define the physics of core collapse supernovae. Electron capture in neutron star crusts affects neutron star cooling. These studies rely strongly on theoretical predictions.

Nuclear theory and society. Last but not least, nuclear physics can and should be used for the benefit of society. The theory roadmap includes theoreti- 
cal advancements relevant for medical applications and for stockpile stewardship. Examples are an ab initio theory for light-ion fusion, a microscopic theory of spontaneous and neutron-induced fission, and reaction theory for medium and heavy nuclei.

Similar to astrophysics, an important stockpile stewardship application involves a complex network of neutron-induced reactions on unstable nuclei that cannot be accessed directly in the laboratory due to the short lifetime of the targets. On the other hand, an indirect approach leading to the same compound nucleus as the desired reaction, such as $(d, p)$, which may be performed in inverse kinematics with radioactive beams at FRIB, can provide important guidance to infer the reaction of interest ${ }^{29}$ However, since this "surrogate" reaction may populate slightly different angular momenta and parities in the entrance channel, a robust theory for nuclear reactions is needed in order to properly infer the results for the desired reaction.

Progress made in the understanding of nuclear reactions within an ab initio framework can provide important information to understand fusion reactions in both astrophysical and terrestrial environments. A recent example concerns uncertainties in the differential cross section for elastic $n-{ }^{3} \mathrm{H}$ scattering, which need to be of the order $5 \%$ to reliably infer a fuel density for inertially confined fusion experiments. ${ }^{\sqrt[30]{0}}$ Theoretical calculations ${ }^{31}$ based on a reaction theory using the $a b$ initio, no-core shell model and the resonating group method ${ }^{\sqrt{32}}$ were able to achieve this accuracy and compare well with data extracted from later experiments ${ }^{33}$ Similar calculations have recently yielded first-principles results for the $d\left({ }^{3} \mathrm{H}, n\right)^{4} \mathrm{He}$ fusion reaction 34 .

\section{Interdisciplinary Aspects of the FRIB Scientific Program}

Physics with exotic nuclei has intimate connections to many research areas outside nuclear structure, reactions, and nuclear astrophysics, see Ref. 35 and Sec. 5 of Ref. 3. Figure 5 illustrates some of these intersections and the shared fundamental questions that tie them together.

A particularly profound synergy exists between research in FRIB physics and research in astrophysics and cosmology. This synergy also ties in to neutrino physics. The near future will see 30-m class optical telescopes and a myriad of new observational probes of the cosmos, from measurements of polarization in the cosmic microwave background (CMB), to 21-cm probes of high redshift $(z \sim 10-100)$, to new X-ray (e.g., NuStar) and Gamma-ray (e.g., Fermi) observatories. Nuclear physics, nuclear astrophysics, and neutrino physics will be key in unraveling what the new data may mean.

As already emphasized, understanding the origin of the elements around us is one of the quests of modern science. The answer lies in understanding the synthesis history of the elements in the universe, and how this evolution gives us insight into the origin and evolution of the structures we see, and the nature of dark matter and dark energy. New data on the history of nucleosynthesis from quite high redshift to 


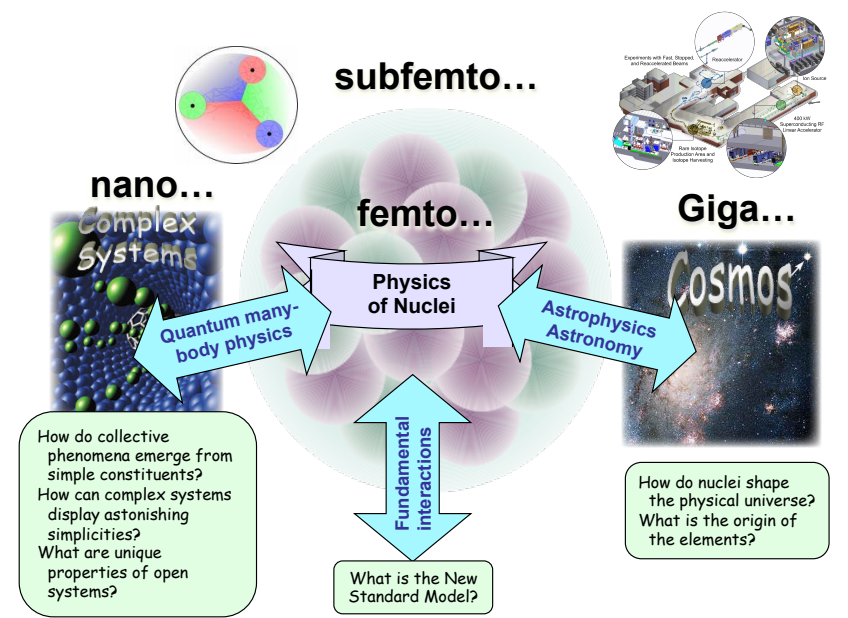

Fig. 5. There are diverse intersections of FRIB science with research in other sub-fields of physics, with shared fundamental questions.

the present epoch will present nuclear astrophysicists with exciting opportunities. At issue is whether the history of nucleosynthesis and star formation is consistent with the picture we have for the mass assembly history of galaxies. Insight into this issue may be key to understanding, for example, the origin and evolution of the dwarf spheroidal galaxies which, in turn, may give insights into the nature of dark matter. 36

CMB observations have given us, or will give us, precise determinations of the baryon density of the universe and the ratio of relativistic to non-relativistic energy density at the epoch of photon decoupling. These, combined with increasingly precise determinations of the primordial deuterium and helium abundances, are creating a nearly over-determined situation for Big Bang Nucleosynthesis (BBN). This turns BBN into an even more powerful probe of the physics of the early universe, particularly of new physics in the neutrino sector, and potentially the QCD epoch. However, there are many open questions involving the synthesis of ${ }^{7} \mathrm{Be}$ and ${ }^{7} \mathrm{Li}$ in BBN (with new physics) and the subsequent fate of these species in stars. These are central problems in nuclear astrophysics. 37

A quantitative understanding of stellar evolution, stellar explosions, and the compact objects they produce, relies on nuclear physics. In the past decade, advances in theory, computation and simulation have helped interpret diverse astrophysical phenomena, unravelling how nuclear physics input shapes observable outcomes. Input from experimental nuclear and neutrino physics has played an important role, but theory is essential to access the enormous range of ambient conditions realized in astrophysics. The nuclear and weak reactions, the equation of state and transport properties of hot and dense matter that play a central role in the most extreme conditions are seldom within reach of direct experiments. A quantitative theory of nuclei and nuclear matter with quantifiable errors and well under- 
stood model systematics can unravel mechanisms that power supernova, gamma-ray bursts, x-ray bursts and neutron star mergers and provide fundamental tests of nuclear physics under extreme conditions. For example, the density dependence of the nuclear symmetry energy influences the spectrum and the time structure of the supernova neutrino signa $\sqrt{38 / 39 / 40}$ and the gravitational wave signal from binary neutron star mergers 41 . The properties of the neutron star crust, where neutronrich nuclei and matter at subnuclear density both play a role, are helping interpret a host of x-ray phenomena associated with accreting neutron stars, and in some cases, permitting us to develop a quantitative theory to predict thermal evolution on timescales of years $\$ \frac{42}{2}$ and motivating a slew of new observations.

There are also numerous profound connections between FRIB science and manybody physics. Despite the fact that the number of nucleons in heavy nuclei is small compared to the number of electrons in solids or atoms in gases, nuclei exhibit emergent phenomena that are present in other complex systems studied by quantum chemists, atomic, molecular, and condensed-matter physicists, and materials scientists. Atomic nuclei exhibit both fundamental and emergent behavior; hence, they provide important clues to our understanding of the transition from microscopic to mesoscopic, and to macroscopic.

One example of fruitful interdisciplinary research that bridges between nano- and femto-scales is the physics of strongly coupled superfluid systems, such as neutron droplets and cold atoms close to the unitary regime: both have been successfully treated by many-body nuclear techniques $\underline{43|44| 45}$ Another example is Cooper pairing. Nucleonic superfluidity lies at the heart of nuclear physics ${ }^{\sqrt{46}}$ it is present in finite nuclei and in the nuclear matter of neutron stars, where spatially anisotropic pairing fields, also discussed in the context of high-temperature superconductivity in novel materials, are expected. Theoretical concepts and tools are shared between the fields. For example techniques developed in nuclear structure physics, such as random matrix theory 47448 and semiclassical methods ${ }^{49}$ have been carried over to the study of mesoscopic systems such as quantum dots and clusters of atoms.

One of the main goals of many-body physics that is shared with nuclear physics is to understand how collective phenomena emerge from simple constituents. We know that complex systems can display astonishing simplicities associated with dynamical many-body symmetries, symmetry breaking effects, and quantum phase transitions, and the atomic nucleus shows many examples of collective behavior. The many-body behavior of neutrinos in a core-collapse supernova, the only manybody system driven by weak interactions, reveals an intriguing connection between neutrinos, emergent properties in many-body physics, and nucleosynthesis.

Open quantum systems, whose properties are affected by the environment of decay, scattering, and reaction channels, are also great interdisciplinary unifiers ${ }^{23}$ Many aspects of open quantum systems that are independent of the system dimensionality are now explored in atomic nuclei, hadrons, molecules, quantum dots and wires and other solid-state microdevices, crystals in laser fields, and microwave cavities. As radioactive nuclear beam experimentation extends the known nuclear 
landscape towards the particle drip lines, the coupling to the continuum space becomes increasingly more important. The novel nuclear approaches developed in this context, such as the continuum shell model $50 \mid 51$ are now being applied to studies of other open quantum systems such as coupled quantum dots or dipole-bound anions.

\section{Enhancing the Feedback Between Experiment and Theory}

The scientific method uses experimentation to assess theoretical predictions. Based on experimental data, the theory is modified and subsequently can be used to guide future measurements. The process is then repeated, until the theory is able to explain observations, and experiment is consistent with theoretical predictions. This positive feedback in the "experiment-theory-experiment-" loop can be enhanced if statistical methods and scientific computing are applied to determine the couplings between model parameters, parameter uncertainties, and the errors of calculated observables.

Nuclei communicate with us through observables revealed by experiment. Some observables are easy to measure; some take considerable effort and experimental ingenuity. Often, the observable, such as the cross section for a certain nuclear reaction channel, can be used to deduce a quantity of interest, like the distribution of neutron matter in the nucleus or the resonance width. A challenge for FRIB science is that in most cases the extraction of structural data will be model dependent. The reliability of the extracted information will depend critically on the ability of theory to accurately describe those reaction processes. Microscopic approaches to reaction theory consistent with the state-of-the-art structure models are necessary to reduce the ambiguity of current highly phenomenological models. Statistical tools will be useful in providing uncertainty quantification. A number of new approaches are being developed for this purpose, but many challenges remain

With nearly 7000 possible isotopes and thousands of pieces of information for each one, not every observable has the potential to impact our understanding in the same way: some measurements are likely to be more important than others. Identification of the key measurements is one way in which theory can provide the foundation for an optimized experimental program at FRIB. By studying the theoretical relevance of the anticipated experimental outcomes, a theoretical assessment of the scientific impact of experiments will help identify critical measurements. However, we also recognize that as with any new facility, surprises not anticipated by current theory are likely to arise. Theory working hand-in-hand with experiment will be key to unravel the puzzles. Past examples of this interplay are the unexpected evolution of shell structure with $N$ and $Z$, and the existence of nuclei with large neutron halos and skins. Often these surprises have provided crucial clues for new physics that was missing from our models.

Theory can also evaluate whether the anticipated experimental errors are adequate to provide meaningful guidance for further model developments. Theory should provide input for planning future experiments by isolating those experimen- 


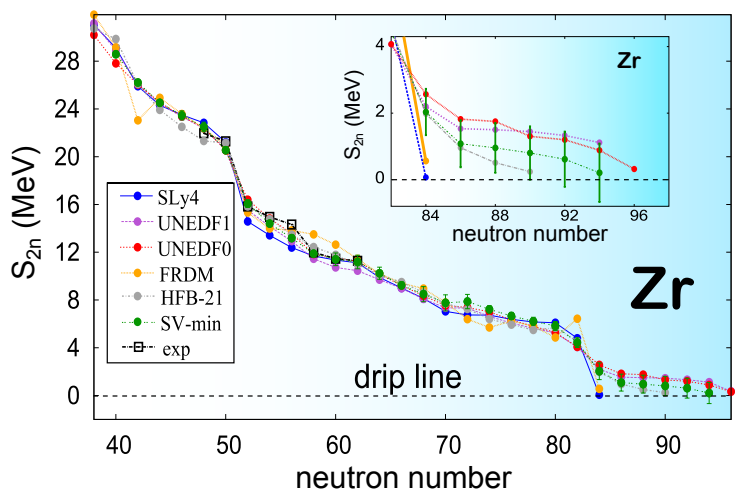

Fig. 6. Calculated and experimental two-neutron separation energies of even-even zirconium isotopes. Calculations performed in Ref. 11 using SLy4, SV-min, UNEDF0, and UNEDF1 energy density functionals are compared to experiment and FRDM ${ }^{53}$ and HFB-2 $1^{\sqrt{54}}$ mass models. The differences between model predictions are small in the region where data (marked by squares) exist and grow steadily when extrapolating towards the two-neutron drip line $\left(S_{2 n}=0\right)$. The bars on the SV-min results indicate statistical errors due to uncertainty in the coupling constants of the functional. Detailed predictions around $S_{2 n}=0$ are illustrated in the inset.

tal data crucial to better constrain nuclear models and validating and verifying model-based extrapolations $\frac{13 \mid 52}{15}$ An FRIB theory effort, working closely with the experimentalists associated with FRIB, will serve as a focal point for facilitating interactions aimed at enhancing the experiment-theory cycle in the scientific method.

By taking advantage of high-performance computing, nuclear theory is developing tools to deliver uncertainty quantification and error analysis for theoretical studies. Statistical tools can also be used to assess the information content of an observable with respect to current theoretical models. Such technologies are essential for providing predictive capability, to estimate uncertainties, and to assess extrapolations - as theoretical models are often applied to entirely new nuclear systems and conditions that are not accessible to experiment. As already discussed in Fig. 3, current nuclear models do not give consistent answers when going outside "safe" regions explored experimentally. Figure 6 illustrates the difficulties encountered with theoretical extrapolations towards drip lines. Shown are the two-neutron separation energies $S_{2 n}$ for the isotopic chain of even-even zirconium isotopes predicted with different theoretical models. In the region for which experimental data are available, all models agree and reproduce the data equally well. However, the discrepancy between various predictions steadily grows when moving away from the region of known nuclei, because the dependence of the effective force on the neutron excess is poorly determined. In the example considered, the neutron drip line is predicted to be between $N=84$ (FRDM and SLy4) and $N=96$ (UNEDF0), i.e., the model (systematic) error is appreciable. In addition to systematic errors, calculated observables are also subject to statistical errors due to uncertainties in model parameters $\frac{55|56| 57}{5 i g u r e} 6$ indicates that the statistical error predicted with the 
SV-min energy density functional gradually grows with $N$. This is primarily caused by the isovector properties of the model that are not well constrained by the current data. For other examples, see Refs. 13, 52, 58, 59, Experimentally, FRIB with its extended reach has the possibility to produce ${ }^{124} \mathrm{Zr}$ and determine its two-neutron separation energy. The figure illustrates that, with the theoretical understanding of the origin of model uncertainties coupled with the new data, a significant improvement in the precision of nuclear models will be possible.

\section{High Performance Computing Aspects of FRIB Science}

As eloquently stated in the recent decadal survey, "High performance computing provides answers to questions that neither experiment nor analytic theory can address; hence, it becomes a third leg supporting the field of nuclear physics." Large-scale nuclear physics computations dramatically increase our understanding of nuclear structure and reactions and the properties of nucleonic matter. A series of workshops on computational physics and forefront areas of nuclear science $\frac{60}{60}$ including QCD, nuclear structure and reactions, and nuclear astrophysics, established the importance and breadth of computational nuclear physics. A large fraction of the discussion revolved around topics critical to FRIB science. The importance of computational nuclear physics has been re-emphasized recently in influential reports 112 Reaching the full potential of the FRIB research program requires comprehensive investigations of many questions that can only be addressed using world-leading computational facilities.

Computational nuclear structure and reactions in the U.S. has advanced significantly through the UNEDF ${ }^{61 \mid 62[63}$ SciDAC project and its successor NUCLEI ${ }^{64}$ Both projects joined forces of nuclear theorists, computer scientists and applied mathematicians to break analytic, algorithmic, and computational barriers in lowenergy nuclear theory. Integral to both projects has been the greatly enhanced degree of quality control: verification of methods and codes, the estimation of uncertainties, and assessment. The UNEDF project helped form a coherent nuclear theory community, opened up new capabilities, fostered transformative science resulting in high-visibility publications, and advanced the careers of many junior scientists. The NUCLEI project encompasses significant components of computational physics relevant to FRIB science; it bridges the scales from hadronic interactions to the structure and dynamics of heavy nuclei to neutron stars within a coherent framework. Figure 7 shows the structure of this project and its ties to applied mathematics and computer science.

The mathematics and computer science components in NUCLEI are directly tied to relevant parts of the NUCLEI project. These groups do forefront research in applied math and computer science that will be both broadly applicable across different fields of science and immediately beneficial to the physics projects in NUCLEI. Examples of such joint projects ${ }^{63}$ include: development of the ADLB (Asynchronous Dynamic Load Balancing) library, which provides scalable load balanc- 


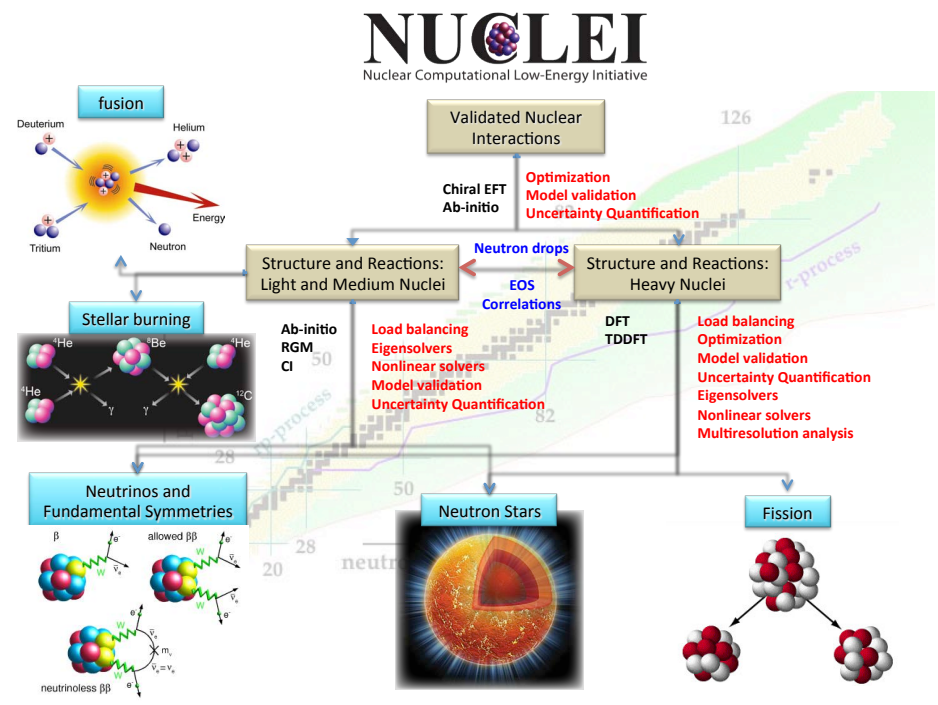

Fig. 7. A schematic diagram of the NUCLEI SciDAC project ${ }^{64}$ showing major efforts in nuclear physics and their ties to applied mathematics and computer science. The NUCLEI project is tied closely to the priorities of the experimental nuclear physics program, including particularly FRIB, but is also important for neutrinoless double beta decay experiments including MAJORANA, EXO, and also to the nuclear physics program with energetic electrons at Jefferson Lab.

ing services for Quantum Monte Carlo calculations on the largest machines available; new schemes for sparse matrix-vector operations and development of efficient scalable iterative eigensolvers for distributed multi-core platforms and topologyaware mapping of computational tasks to reduce communication overhead employed in state-of-the-art CI calculations; applications of the wavelet-based MADNESS (Multiresolution Adaptive Numerical Environment for Scientific Simulations) to nuclear DFT; derivative-free multi-parameter optimizations of chiral interactions and energy density functionals with Practical Optimization Using No DERivatives (POUNDERS) framework; and uncertainty quantification using the Gaussian Process Models for Simulation Analysis tool, which is important in many applications from nuclear interaction input to the significance of predictions to upcoming experiments.

Many scientific advances in the field were made possible by a rapid increase in our ability to use the largest-scale computational resources. Large-scale usage in nuclear structure and reactions in the U.S. rose from $80 \mathrm{M}$ core-hr/year in 2009 to $320 \mathrm{M}$ core-hr/year in 2013. In the future, our ability to use the largest-scale computers will require additional investments in manpower, particularly as we transition to new architectures. These new positions are critical to enable the effort to scale to the largest-scale machines heading toward exascale, and to support the FRIB-related experimental programs in a timely manner.

There are also deep connections between NUCLEI and computational efforts in 
neighboring areas. At the shortest-scales, lattice gauge theory plays an important role, particularly for aspects such as the three-neutron force that are difficult to isolate, and often access, experimentally. These can play a very important role in neutron-rich nuclei and matter. Simulations of neutron star structure and explosive events on neutron stars and neutron star mergers will yield critical information about dense matter, as decribed in Sec. 2. Supernovae simulations encompass important studies of radiation hydrodynamics that illuminate the explosion mechanism and nucleosynthesis, to studies of neutrino propagation that can tell us more about the astrophysics of supernovae as well as neutrino properties. Examples of such simulations are displayed in Fig. 2, Every one of the research areas displayed in Figs. 114 and 6 require state-of-the art computational facilities and teams of physicists, computer scientists, and applied mathematicians working together to advance our understanding and to fully exploit the capabilities of FRIB.

\section{FRIB Theoretical Science Organization}

The current nuclear theory effort in the U.S. related to FRIB is quite broad and dispersed, with large groups at National Laboratories and some universities, but also many small university groups. There are theorists studying astrophysical phenomena, nuclear reactions, nuclear equations of state, nuclear structure, and nuclear applications in national security and isotope R\&D. Some function largely independently while others collaborate in large research projects such as the NUCLEI effort discussed in Sec. 5 or topical collaborations in nuclear theory on neutrinos and nucleosynthesis in hot and dense matter ${ }^{65}$ and reactions for unstable isotopes. $\underline{66}$

The theory community interested in FRIB physics formed, over a decade ago, the RIA ("Rare Isotope Accelerator") and FRIB theory users groups, whose primary purpose is to identify and prioritize the most important theory developments in relation to RIA and FRIB projects, to advocate for the science of radioactive beams, and to be a voice for the low-energy nuclear theory and astrophysics community. The members of the FRIB Theory Users Group regularly join the annual low-energy nuclear physics community meetings. During the last 10 years, the theory community has produced two important documents: the RIA Theory Bluebook $(2005)^{67}$ and an FRIB Theory Users Group Report (2011) ${ }^{[68}$ The former report outlined various scientific directions necessary for an impactful theory effort, while the latter focused on issues surrounding education and training of the next generation of theorists, and included results from a survey on the needs of the field. While the 2011 survey is new from a time perspective, we note that the NSAC theory report from $2003^{69}$ also recommended ways to address pipeline issues.

To address the concerns discussed in the 2011 report, the pipeline of young theorists must be maintained. In particular, post-doctoral appointments are crucial for R\&D career development. An national FRIB Theory Fellow program would address the problem, creating opportunities for young post-doctoral fellows to mature and fostering continuous interactions among theorists and experimentalists. The need 
for faculty or laboratory positions in nuclear theory has also been noted in the past, and was the second concern documented in Ref. 68. To overcome this problem, a bridging program for young faculty could be developed enabling needed growth into areas of critical need.

The nuclear theory community in the U.S. has also extended beyond its boundaries. The report from a recent comparative research review of nuclear physics for the U.S. Department of Energy ${ }^{70}$ states: "New RIB facilities are under construction in Canada, France, and Asia. With the wider spread of world-leading experimental facilities, international networking will become more important in the future. As a first step, DOE has created exchange programs for nuclear theorists with selected countries. These programs should be expanded and include joint graduate education with international partners." There are a number of initiatives already in place, that foment collaborations between countries, namely JUSTIPEN ${ }^{71}$, FUSTIPEN ${ }^{72}$, and CUSTIPEN 73 , which are theory exchange programs. In addition, International Collaborations in Nuclear Theory ${ }^{\sqrt{74}}$ coordinates theory topical programs between NSCL/FRIB, GSI and RIKEN, to address theoretical issues relevant to those laboratories. Finally, the TALENT initiative in graduate education, discussed in Sec. 7 below, has also spurted from an international framework.

\section{Education Challenges}

The theory effort around FRIB will also play an important role in the development of a broad, modern, and attractive educational curriculum addressing the nuclear many-body problem and related areas. A thorough knowledge of up-to-date theoretical methods and phenomenology will be required to tackle the theoretical and experimental challenges that will be faced by the next generation of nuclear physicists working in FRIB science. However, as discussed in Sec. 6. most university low-energy nuclear theory groups are small and, therefore, unable to offer a broad spectrum of advanced research-based nuclear physics courses. Fortunately, with advances in modern educational and computational tools, we are in a situation where globally coordinated efforts can make a significant qualitative difference in the way nuclear physics students are educated.

Recently, nuclear physicists in North America and Europe have teamed up to launch an educational initiative dubbed Nuclear TALENT (Training in Advanced Low-Energy Nuclear Theory) ${ }^{75}$ The long-term vision of TALENT is to develop a coherent graduate curriculum that will provide the foundations for a cross-cutting low-energy nuclear theory research program, and will link modern theoretical approaches with on-going experimental efforts. To meet these objectives, educational modules are being commissioned from the best teachers and specialists in low-energy nuclear theory. The resulting unique material is being collected in the form of webbased courses, books, and other educational resources. The development of such a knowledge base will allow highly specialized university groups to benefit greatly from a broad selection of topics taught by world-leading experts. 
In its initial phase, TALENT has selected several topics in low-energy nuclear physics for teaching modules. The range of courses is broad, from nuclear forces and $a b$ initio approaches, to the theory of complex nuclei, to nuclear reactions and open quantum systems, to nuclear astrophysics, to computational tools for nuclear physics. Some of the topics have already been taught as intensive threeweek courses hosted by the European Centre for Theoretical Studies in Nuclear Physics and Related Areas in Trento, the National Institute for Nuclear Theory in Seattle, and GANIL in Caen. Other courses are in the pipeline, including one at the Joint Institute for Nuclear Astrophysics.

One of the major challenges facing TALENT is to develop a robust model for funding. To this point, all courses have been run on a voluntary basis, with financial support for students (lodging and local expenses) provided by the hosting institutions. Obviously, to put this educational initiative on solid ground, sustainable funding is needed. In addition, a proper model for transferring academic credit must be developed. There exist examples of educational collaborations between various universities in the U.S. where bilateral agreements have been developed between various colleges of natural science, enabling an economically sustainable model for credit transfers. With such bilateral agreements, TALENT courses can be included in course curricula of participating institutions, and teaching duties could also be transferred. With an FRIB theory center on the horizon, there are good prospects for a better coordination of educational efforts in advanced theory of nuclei and nucleonic matter. While the current TALENT effort is built around theoretical and experimental activities in North America and Europe, its expansion into other regions is envisioned. In particular, considering the scale of experimental efforts in rare-isotope science in Japan and China, it is anticipated and hoped that Asia will soon join the initiative.

\section{Summary}

An understanding of the properties of atomic nuclei and their reactions is essential for a complete description of nuclei, an explanation of element formation and the properties of stars, and for present and future energy, defense, and security applications. This requires a coherent picture across many energy scales, all the way from the interactions between nucleons to the superheavy nuclei and neutron stars.

The roadmap for low-energy nuclear theory is well established $61|62| 67$ It involves the extension of $a b$ initio and configuration interaction approaches all the way to medium-heavy nuclei, and the quest for a universal nuclear density functional that will allow description of all nuclei up to the heaviest elements and neutron stars. It also includes developments in reaction theory required for a meaningful link to experiment. The direct coupling from nucleon-nucleon interaction scales $(\sim 100 \mathrm{MeV})$ to nuclear binding scales $(\sim 1-10 \mathrm{MeV})$ to collective excitation scales $(<1 \mathrm{MeV})$, facilitated by effective field theory, provides a coherent picture of the structure and dynamics of all nuclei and nucleonic matter found in astrophysical environments. 
To realize this vision, the properties of rare isotopes are an essential guide.

The development of a theoretical framework that connects the light and heavy nuclei, proton-rich and neutron-rich rare isotopes, and dense neutron matter is within reach. In the next decade, these developments will have profound implications for nuclear physics, nuclear astrophysics, and neighboring areas, such as high-energy astrophysics, fundamental interaction physics, and hadron structure.

With FRIB, the field has a clear path to achieve its overall scientific goals and to answer the overarching questions. With FRIB, we will have the ability to produce the key isotopes now unavailable. FRIB will be the world's most powerful facility to explore the rare-isotope frontier, making nearly $80 \%$ of the isotopes predicted to exist for elements up to uranium and providing access to beams of the most interesting isotopes. By taking advantage of the unique coupling of crucial data from FRIB and other radioactive beam facilities with advanced theoretical frameworks and high-performance computing, nuclear theory will be able to develop a predictive picture of nucleonic matter. This is an exciting perspective.

\section{Acknowledgments}

This work was supported by the U.S. Department of Energy under Contract No. DE-FG02-96ER40963 (University of Tennessee), Nos. de-sc0008499/DE-SC0008533 (NUCLEI SciDAC Collaboration), DE-AC02-06CH11357 (Argonne National Laboratory), DE-AC52-07NA27344 (Lawrence Livermore National Laboratory), DEAC52-06NA25396 (Los Alamos National Laboratory), de-sc0004087 and DE-FG5208NA28552 (Michigan State Univ), and DE-AC05-00OR22725 (Oak Ridge National Laboratory); the National Science Foundation under Grant No. PHY-1306250 (Ohio State University), PHY-1068022 (Texas A\&M University-Commerce), PHY1068271 (Michigan State Univ), PHY-0970064 and PHY-1307372 (University of California, San Diego), PHY-1205024 (University of Wisconsin-Madison); and the National Aeronautics and Space Administration under grant NNX11AC41G issued through the Science Mission Directorate (Texas A\&M University-Commerce).

\section{References}

1. Nuclear Physics: Exploring the Heart of Matter. Report of the Committee on the Assessment of and Outlook for Nuclear Physics (The National Academies Press, 2012).

2. Report to NSAC on Implementing the 2007 Long Range Plan (2013).

3. The Frontiers of Nuclear Science: A Long Range Plan NSAC Report (2007).

4. Scientific Opportunities with a Rare-Isotope Facility in the United States. Report of the NAS/NRC Rare Isotope Science Assessment Committee (The National Academies Press, 2007).

5. G. Bollen, AIP Conf. Proc. 1224, 432 (2010).

6. NSAC Rare-Isotope Beam (RIB) Task Force Report (2007).

7. D. Morrissey and B. Sherrill, The Euroschool Lectures on Physics with Exotic Beams, Vol. 1 (Springer-Verlag, 2004).

8. J. Wei et al., in Proceedings of 12th Heavy Ion Accelerator Technology Conference; Chicago, IL USA, (2012). p. 8. 
9. J. Wei et al., in Proceedings of the North American Particle Accelerator Conference; Pasadena, CA, USA, (2013).

10. D. Geesaman, C. Gelbke, R. Janssens and B. Sherrill, Ann. Rev. Nucl. Part. Sci. 56, 53 (2006).

11. J. Erler, N. Birge, M. Kortelainen, W. Nazarewicz, E. Olsen, A. Perhac and M. Stoitsov, Nature 486, 509 (2012).

12. FRIB Estimated Rates: http://groups.nscl.msu.edu/frib/rates/.

13. M. Kortelainen, J. Erler, W. Nazarewicz, N. Birge, Y. Gao and E. Olsen, Phys. Rev. C 88, 031305 (2013).

14. Proceedings of the 2012 Workshop on Isotope Harvesting at FRIB, East Lansing, MI July 2012.

15. J. Engel, M. J. Ramsey-Musolf and U. van Kolck, Prog. Part. Nucl. Phys. 71, 21 (2013).

16. H. Schatz, Physics Today 61, 40 (2008).

17. S. Bruenn et al., ApJ 767, L6 (2012).

18. S. Rosswog, T. Piran and E. Nakar, MNRAS 430, 2585 (2013).

19. M. Wiescher, F. Käppeler and K. Langanke, ARA\&A 50, 165 (2012).

20. A. T. Gallant et al., Phys. Rev. Lett. 109, 032506 (2012).

21. F. Wienholtz et al., Nature 498, 346 (2013).

22. D. Steppenbeck et al., Nature 502, 207 (2013).

23. N. Michel, W. Nazarewicz, J. Okołowicz and M. Płoszajczak, J. Phys. G 37, 064042 (2010).

24. J. M. Lattimer and Y. Lim, ApJ 771, 51 (2013).

25. S. Gandolfi, J. Carlson and S. Reddy, Phys. Rev. C 85, 032801 (2012).

26. B.-A. Li and X. Han, Phys. Lett. B 727, 276 (2013).

27. P. B. Demorest, T. Pennucci, S. M. Ransom, M. S. E. Roberts and J. W. T. Hessels, Nature 467, 209 (2010).

28. J. Antoniadis et al., Science $\mathbf{3 4 0}$ (2013).

29. J. E. Escher, J. T. Burke, F. S. Dietrich, N. D. Scielzo, I. J. Thompson and W. Younes, Rev. Mod. Phys. 84, 353 (2012).

30. J. A. Frenje et al., Phys. Plasmas 17, 056311 (2010).

31. P. Navratil, S. Quaglioni and R. Roth, J. Phys. Conf. Ser. 312, 082002 (2011).

32. S. Quaglioni and P. Navratil, Phys. Rev. C 79, 044606 (2009).

33. J. A. Frenje et al., Phys. Rev. Lett. 107, 122502 (2011).

34. P. Navratil and S. Quaglioni, Phys. Rev. Lett. 108, 042503 (2012).

35. The Intellectual Challenges of RIA: A White Paper from the RIA Users Community (2002).

36. M. Boylan-Kolchin, J. S. Bullock and M. Kaplinghat, MNRAS 422, 1203 (2012).

37. A. B. Balantekin and G. M. Fuller, Prog. Part. Nucl. Phys. 71, 162 (2013).

38. L. F. Roberts, G. Shen, V. Cirigliano, J. A. Pons, S. Reddy and S. E. Woosley, Phys. Rev. Lett. 108, 061103 (2012).

39. L. F. Roberts, S. Reddy and G. Shen, Phys. Rev. C 86, 065803 (2012).

40. G. Martinez-Pinedo, T. Fischer, A. Lohs and L. Huther, Phys. Rev. Lett. 109, 251104 (2012).

41. A. Bauswein and H.-T. Janka, Phys. Rev. Lett. 108, 011101 (2012).

42. D. Page and S. Reddy, Phys. Rev. Lett. 111, 241102 (2013).

43. A. Gezerlis and J. Carlson, Phys. Rev. C 77, 032801 (2008).

44. N. T. Zinner and A. S. Jensen, J. Phys. G 40, 053101 (2013).

45. H.-W. Hammer, A. Nogga and A. Schwenk, Rev. Mod. Phys. 85, 197 (2013).

46. R. A. Broglia and V. Zelevinsky (eds.), Fifty Years of Nuclear BCS (World Scientific, 
2012).

47. T. Papenbrock and H. A. Weidenmüller, Rev. Mod. Phys. 79, 997 (2007).

48. V. Zelevinsky, J. Phys. G 37, 064024 (2010).

49. A. Magner, I. Yatsyshyn, K. Arita and M. Brack, Phys. At. Nucl. 74, 1445 (2011).

50. N. Michel, W. Nazarewicz, M. Płoszajczak and T. Vertse, J. Phys. G: Nucl. Part. Phys. 36, 013101 (2009).

51. G. Papadimitriou, J. Rotureau, N. Michel, M. Płoszajczak and B. R. Barrett, Phys. Rev. C 88, 044318 (2013).

52. J. Piekarewicz, B. K. Agrawal, G. Colò, W. Nazarewicz, N. Paar, P.-G. Reinhard, X. Roca-Maza and D. Vretenar, Phys. Rev. C 85, 041302 (2012).

53. P. Möller, J. Nix, W. Myers and W. Swiatecki, At. Data Nucl. Data Tables 59, 185 (1995).

54. S. Goriely, M. Samyn, M. Bender and J. M. Pearson, Phys. Rev. C 82, 035804 (2010).

55. P. Klüpfel, P.-G. Reinhard, T. J. Bürvenich and J. A. Maruhn, Phys. Rev. C 79, 034310 (2009).

56. P.-G. Reinhard and W. Nazarewicz, Phys. Rev. C 81, 051303 (2010).

57. F. J. Fattoyev and J. Piekarewicz, Phys. Rev. C 84, 064302 (2011).

58. A. Afanasjev, S. Agbemava, D. Ray and P. Ring, Phys. Lett. B 726, 680 (2013).

59. J. Erler, C. J. Horowitz, W. Nazarewicz, M. Rafalski and P.-G. Reinhard, Phys. Rev. C 87, 044320 (2013).

60. Scientific Grand Challenges Workshop Series, http://science.energy.gov/ascr/newsand-resources/workshops-and-conferences/grand-challenges/.

61. G. F. Bertsch, D. J. Dean and W. Nazarewicz, SciDAC Review 6, 42 (2007).

62. R. Furnstahl, Nucl. Phys. News 21, 18 (2011).

63. S. Bogner et al., Comput. Phys. Commun. 184, 2235 (2013).

64. Nuclear Computational Low-Energy Initiative, http://computingnuclei.org.

65. Topical collaboration on neutrinos and nucleosynthesis in hot and dense matter, https://sites.google.com/site/doetopicalnnhdm/.

66. Topical collaboration on Theory of Reactions for Unstable iSotopes, http://www.reactiontheory.org.

67. RIA Theory Bluebook: A Road Map. A Report from the RIA Theory Group, $200 \%$.

68. A. B. Balantekin, R. H. Cyburt, W. C. Haxton, W. Nazarewicz, F. Nunes, T. Papenbrock, S. Pratt and J. Vary, FRIB Theory Users Group Report: Joint ATLASHRIBF-NSCL-FRIB SuperUsers Meeting, August 2011; arXiv:nucl-th/1109.5787.

69. J. Carlson, B. Holstein, X. Ji, G. McLaughlin, B. Müller, W. Nazarewicz, K. Rajagopal, W. Roberts and X.-N. Wang, A Vision for Nuclear Theory: Report to NSAC (2003); arXiv:nucl-th/0311056

70. Nuclear Physics Comparative Research Review for the U. S. Department of Energy (2013).

71. JUSTIPEN website, http://www.phys.utk.edu/justipen/.

72. FUSTIPEN website, http://fustipen.ganil.fr.

73. CUSTIPEN website, http://custipen.pku.edu.cn/index.html.

74. ICNT, http://frib.msu.edu/content/international-collaborations-nuclear-theory-icnt.

75. TALENT website, http://nucleartalent.org. 